\title{
Investigations of the measurement accuracy of stereo particle image velocimetry
}

\author{
R. Fei, W. Merzkirch
}

Abstract With a stereo PIV system, in order to perform reliable measurements of the three velocity components in liquid flow, it is mandatory to minimise the errors made in determining the $2 \mathrm{D}$ displacement vectors and the viewing direction of each of the two cameras. We present a method for determining the viewing direction in the "angular displacement" stereo system by means of a digital imaging procedure such that the direct measurement of geometrical parameters of the set-up is avoided. This makes the method particularly useful for measurements through the transparent walls confining the liquid flow. A third order polynomial used for calibrating the stereo system is shown to provide more accurate results than imaging functions of lower order. Further improvement of the evaluation accuracy is obtained with the application of an artificial neuronal network, but at the expense of considerably increasing the computation time. A comparison of the evaluation results obtained with the operational procedures presented in this paper with those generated with another method that is applicable to liquid flow (Soloff et al. 1997) shows, that the present procedures can be considered as a viable alternative to existing methods.

\section{1}

\section{Introduction}

During the past decade the PIV technology has been extended towards stereo systems which allow to measure the out-of-plane velocity component together with the two components in the plane of the laser light sheet. Stereo PIV systems use two cameras that record the two-dimensional (2D) in-plane particle image patterns under different viewing directions. Currently, the "angular displacement" system is the standard configuration of the two cameras, and fulfilling the Scheimpflug condition ensures that all particle images are focused (Prasad 2000; Raffel et al. 1998; Hinsch 1995).

The three-component (3C) velocity vectors are determined from the two separate two-component (2C) vector patterns by means of a special reconstruction algorithm. It

Received: 12 November 2003 / Accepted: 10 June 2004

Published online: 20 July 2004

(C) Springer-Verlag 2004

R. Fei, W. Merzkirch ( $\square)$

Strömungslehre, Universität Essen, 44797 Essen,

Germany

E-mail: wolfgang.merzkirch@uni-essen.de is reported that the error in the out-of-plane component relative to the in-plane component is equal to $1 / \tan \alpha$, where $\alpha$ is the viewing angle (see, e.g. Prasad 2000; Lawson and Wu 1997; Prasad and Jensen 1995; Prasad and Adrian 1993). The error in determining the out-ofplane component is composed of the errors made in measuring the two $2 \mathrm{C}$ velocity fields and the error involved in the reconstruction process. Minimising this error is still the aim of current research (see, e.g. Coudert and Schon 2001).

Calculating the three Cartesian components of the particle displacement, $\mathrm{d} x, \mathrm{~d} y, \mathrm{~d} z$, from the in-plane displacements $\mathrm{d} x_{1}, \mathrm{~d} y_{1}, \mathrm{~d} x_{2}, \mathrm{~d} y_{2}$ (the indices 1,2 refer to cameras 1 and 2, respectively; see Fig. 1) can be done by means of the following equations:

$$
\begin{aligned}
\mathrm{d} x & =\frac{\mathrm{d} x_{2} \cdot \tan \alpha_{1}-\mathrm{d} x_{1} \cdot \tan \alpha_{2}}{\tan \alpha_{1}-\tan \alpha_{2}} \\
\mathrm{~d} y & =\frac{1}{2}\left(\mathrm{~d} y_{1}+\mathrm{d} y_{2}\right) \\
\mathrm{d} z & =\frac{\mathrm{d} x_{1}-\mathrm{d} x_{2}}{\tan \alpha_{1}-\tan \alpha_{2}}
\end{aligned}
$$

$\alpha_{1}, \alpha_{2}$ define the respective viewing directions with $\alpha_{1}>0^{\circ}$ and $\alpha_{2}<0^{\circ}$ (angle between optical axis of camera and direction normal to light sheet plane $z$ ). The symbols used in Eq. 1 are explained in Fig. 1. The first and third relationships are almost identical with Eqs. 1 and 3 in Willert (1997); they differ in comparison to Willert by the sign on the right side of Willert's Eq. 3 because the viewing angles $\alpha_{i}$ are defined in a different way. The second relationship applies to very small values of $\beta_{i}$ as it is also shown in Willert (1997); this simplification is justified because the two cameras in the set-up considered here are situated in a horizontal plane $\left(\beta_{i}<<0\right)$. Details of the derivation of Eq. 1 can also be found in Fei (2002).

$\mathrm{d} X$ and $\mathrm{d} Y$ are projections of the particle displacement on the $x-z$ and $y-z$ planes, respectively. The quantities $\mathrm{d} x_{i}$, $\mathrm{d} y_{i}, \alpha_{i}$ must be measured with the highest achievable accuracy. It is known that, in the "angular displacement" system, the imaging ratio is not constant across the whole field of each 2D PIV recording. Therefore, a necessary first step should correct the distorted 2D PIV recordings by means of an appropriate transformation, such that the displacements $\mathrm{d} x_{i}, \mathrm{~d} y_{i}$ are the "correct" values in the transformed $x-y$ coordinate systems. Different calibration procedures using imaging functions or mapping algorithms have been reported for this purpose (e.g. Ehrenfried 2002; Westerweel and van Oord 1999; Jähne 1997; Willert 1997). In this paper we investigate and 

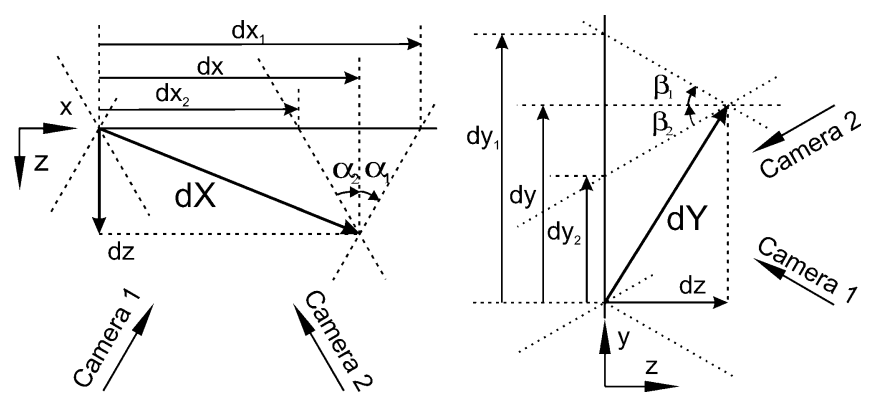

Fig. 1. Definition of geometrical quantities used for describing the "angular displacement" stereo PIV system

discuss a number of operational procedures that can be considered as alternatives for known procedures used in practical stereo PIV systems. These have a third order polynomial as the imaging function, and a method based on the use of an artificial neuronal network (ANN) for the same purpose. Furthermore, we propose a procedure for determining the angular directions $\alpha_{i}$ by means of digital image processing. We emphasise possible applications of the proposed procedures to liquid flows where the imaging must be done through one or several glass plates. The questions we would like to answer are: Which accuracy can be achieved when these procedures are used in a measurement system, and at what expense.

The proposed operational procedures are demonstrated in an experiment and compared with an existing method. For the PIV evaluation, i.e. the measurement of the particle displacements, we use the MQD method (Gui and Merzkirch 1996) that, for the present applications, has been proven to ensure a high evaluation accuracy (Fei et al. 1999). Details of the derivation of the new procedures and experimental verification can be found in the dissertation of Fei (2002).

\section{2}

\section{Determining the viewing direction}

The reconstruction of the $3 \mathrm{D}$ velocity or displacement vector according to Eq. 1 requires knowledge of the viewing direction, expressed by the angles $\alpha_{1}, \alpha_{2}$. In many cases it is not possible to determine $\alpha_{1}$ and $\alpha_{2}$ from the known geometry of the set-up with high precision, because the distance between light sheet and image plane of the cameras cannot be measured with sufficient accuracy, particularly if the imaging takes place through transparent walls. Before describing a new procedure for determining $\alpha_{1}$ and $\alpha_{2}$, we shall give an estimate of the influence of the error made when determining the viewing angle $\mathrm{d} \alpha$ on the inaccuracy of the displacement component $\mathrm{d} z$.

In order to facilitate the derivation and form of the following equations (Eqs. 2 and 3) we assume a symmetrical orientation of the two cameras with $\left|\alpha_{1}\right|=\left|\alpha_{2}\right|$. This assumption was not used for determining the viewing angle (Eq. 4), so that the two viewing angles can be determined independently of each other. Therefore, only one camera is considered and depicted in the respective figure (Fig. 2). From Eq. 1 and with $\left|\alpha_{1}\right|=\left|\alpha_{2}\right|=\alpha$ one derives for the differential of $\mathrm{d} z$

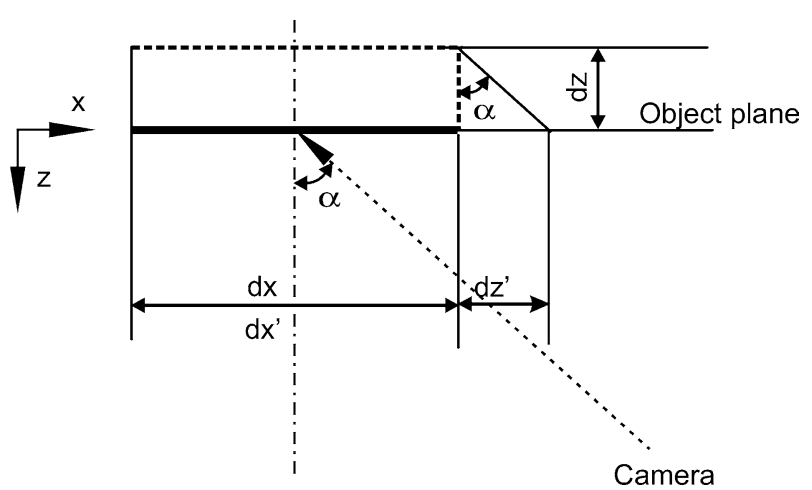

Fig. 2. Determination of the viewing angle $\alpha$. Typical dimensions in the experiments are $\mathrm{d} x=5 \mathrm{~mm}, \mathrm{~d} z=0.5-1.0 \mathrm{~mm}$

$$
\delta(\mathrm{d} z)=\frac{1}{2} \frac{1}{\tan \alpha} \cdot \delta\left(\mathrm{d} x_{1}-\mathrm{d} x_{2}\right)+\frac{1}{2}\left(\mathrm{~d} x_{1}-\mathrm{d} x_{2}\right) \cdot \delta\left(\frac{1}{\tan \alpha}\right)
$$

The relative deviation of $\mathrm{d} z$

$$
\delta(\mathrm{d} z) / \mathrm{d} z=\frac{\delta\left(\mathrm{d} x_{1}-\mathrm{d} x_{2}\right)}{\mathrm{d} x_{1}-\mathrm{d} x_{2}}-\frac{1}{\sin \alpha \cdot \cos \alpha} \cdot \delta \alpha
$$

is composed of two contributions: The first term on the left side describes the error made in the 2D PIV recordings, the second term is the contribution due to the inaccuracy of determining the viewing direction. It can be seen that $\delta(\mathrm{d} z) / \mathrm{d} z$ increases with decreasing value of $\alpha$, and for typical number values of $\alpha$ one can derive that a relative error of a few percent in $\alpha$ may cause relative deviations in $\mathrm{d} z$ of also a few percent. This calls for a precise method for determining $\alpha$.

For the proposed method of determining the viewing angle $\alpha$ a reference scale of length $\mathrm{d} x$ is placed in the object plane. The procedure is explained with reference to the geometry defined in Fig. 2. The scale that is parallel to the light sheet or object plane is displaced normal to that plane by the amount $\mathrm{d} z$. The projection of $\mathrm{d} z$ onto the object plane is $\mathrm{d} z^{\prime}=\mathrm{d} z \cdot \tan \alpha$, that of $\mathrm{d} x$ is $\mathrm{d} x^{\prime}$. If the imaging ratio is $M$, the projections are $\mathrm{d} x_{\mathrm{m}}^{\prime}=M \cdot \mathrm{d} x^{\prime}$ and $\mathrm{d} z_{\mathrm{m}}^{\prime}=M \cdot \mathrm{d} z^{\prime}$, respectively. Then, the viewing angle $\alpha$ can be determined from

$\tan \alpha=\frac{\mathrm{d} z_{\mathrm{m}}^{\prime}}{\mathrm{d} z} \cdot \frac{\mathrm{d} x}{\mathrm{~d} x_{\mathrm{m}}^{\prime}}$

It is known that in the "angular displacement" system the imaging ratio is not constant across the whole field of each 2D PIV recording. An imaging function (described next) was used to convert the image recorded with distortion into an "ideal" new image free of distortion with a constant imaging ratio $M$ and the same value of the viewing angle within the whole image, before the viewing angle was calculated.

In a calibration $\mathrm{d} x$ and $\mathrm{d} z$ should be determined with high accuracy; $\mathrm{d} x_{\mathrm{m}}^{\prime}$ and $\mathrm{d} z_{\mathrm{m}}^{\prime}$ are measured in the (digital) imaging plane. For determining $\mathrm{d} z^{\prime}{ }_{\mathrm{m}}$, two exposures, taken before and after displacing the object plane by $\mathrm{d} z$, are correlated, and $\mathrm{d} z^{\prime}{ }_{\mathrm{m}}$ can be measured with sub-pixel accuracy. It is thus unnecessary to measure any geomet- 
rical parameter of the experimental set-up, so that this procedure accounts automatically for the imaging through transparent walls when the stereo system is applied to liquid flow confined by such walls. It is difficult to give an estimate of the error made in determining the viewing angle in this way, because we have no means to determine a reference value of $\alpha$ for the arrangement in which the object is situated in a water tank and seen through (at least) one glass window. We can only use the result of the experiment described in Sect. 4 as an indirect proof for the accuracy of the method.

\section{3}

\section{Imaging function}

\section{1}

\section{Third order polynomial}

The imaging function serves to transform a recorded image into a different coordinate system in order to compensate for distortions. The transformed image is called the physical plane. Here, the aim is that the transformed image exhibits a constant imaging ratio throughout the whole physical plane. For this purpose we use a third order polynomial of the following form:

$$
\begin{aligned}
x_{i}= & a_{1} X_{i}^{3}+a_{2} Y_{i}^{3}+a_{3} X_{i}^{2} Y_{i}+a_{4} X_{i} Y_{i}^{2}+a_{5} X_{i}^{2}+a_{6} Y_{i}^{2} \\
& +a_{7} X_{i} Y_{i}+a_{8} X_{i}+a_{9} Y_{i}+a_{10} y_{i} \\
= & b_{1} X_{i}^{3}+b_{2} Y_{i}^{3}+b_{3} X_{i}^{2} Y_{i}+b_{4} X_{i} Y_{i}^{2}+b_{5} X_{i}^{2}+b_{6} Y_{i}^{2} \\
& +b_{7} X_{i} Y_{i}+b_{8} X_{i}+b_{9} Y_{i}+b_{10}
\end{aligned}
$$

$x_{i}, y_{i}$ are the coordinates in the physical plane, while $X_{i}$, $Y_{i}$ are the coordinates in the recording plane. For determining the 20 constant coefficients $a_{1} \ldots a_{10}$ and $b_{1} \ldots b_{10}$, it is required that the coordinates $x_{i}, y_{i}, X_{i}, Y_{i}$ are known for $m$ points in the two planes with $m \geq 10$. A scheme for solving the system for the 20 unknowns by means of a set of $2 \mathrm{~m}$ linear equations is included in Fei (2002). The identification of the $m$ points in the recording plane and the physical plane is done in a calibration process, for which a calibration grid is placed in the light sheet plane. The procedure is similar to methods described by, e.g. Ehrenfried (2002); Westerweel and van Oord (1999); Willert (1997).

\section{2}

\section{Comparative study of different imaging functions}

As mentioned above, a number of imaging functions or mapping procedures have been reported in the literature. Here, we compare three algebraic functions and the third order polynomial according to Eq. 5 by applying these relationships to mapping a calibration grid that is placed in the centre of the light sheet in a water tank. The imaging is done through the transparent glass walls of the tank (for dimensions, see Sect. 4.1). The arrangement for these studies is the same as sketched in Fig. 7 (see Sect. 4.2). The rectangular grid has a total of $9 \cdot 13=117$ nodal points. Of these points, 93 serve as reference coordinates for determining the constants used in the imaging functions $(m=93)$. The coordinates of the remaining 24 points in the physical plane are determined by means of the imaging functions. Since the true coordinates of these points are known, the accuracy of the mapping procedure can be determined.

The following imaging functions from the literature are used for this comparison:

a) The second order polynomial reported by Westerweel and van Oord (1999)

$x_{i}=a_{1} X_{i}^{2}+a_{2} Y_{i}^{2}+a_{3} X_{i} Y_{i}+a_{4} X_{i}+a_{5} Y_{i}+a_{6}$

$y_{i}=b_{1} X_{i}^{2}+b_{2} Y_{i}^{2}+b_{3} X_{i} Y_{i}+b_{4} X_{i}+b_{5} Y_{i}+b_{6}$

b) The (first order) mapping function defined by Willert (1997)

$x_{i}=\frac{a_{11} X_{i}+a_{12} Y_{i}+a_{13}}{a_{31} X_{i}+a_{32} Y_{i}+1}$

$y_{i}=\frac{a_{21} X_{i}+a_{22} Y_{i}+a_{23}}{a_{31} X_{i}+a_{32} Y_{i}+1}$

c) The (second order) mapping function defined by Willert (1997)

$x_{i}=\frac{a_{11} X_{i}^{2}+a_{12} Y_{i}^{2}+a_{13} X_{i} Y_{i}+a_{14} X_{i}+a_{15} Y_{i}+a_{16}}{a_{31} X_{i}^{2}+a_{32} Y_{i}^{2}+a_{33} X_{i} Y_{i}+a_{34} X_{i}+a_{35} Y_{i}+1}$

$y_{i}=\frac{a_{21} X_{i}^{2}+a_{22} Y_{i}^{2}+a_{23} X_{i} Y_{i}+a_{24} X_{i}+a_{25} Y_{i}+a_{26}}{a_{31} X_{i}^{2}+a_{32} Y_{i}^{2}+a_{33} X_{i} Y_{i}+a_{34} X_{i}+a_{35} Y_{i}+1}$

The grid was shifted to a number of $z$-positions in the light sheet within an interval of $\Delta= \pm 0.5 \mathrm{~mm}$, and at each position the procedure was performed for the 24 test points. The RMS values of the deviation from the true coordinates (in pixels) found for the 24 points at different $z$-positions (abscissa) are given in Fig. 3. The smallest deviation is obtained when applying the third order polynomial, Eq. 5. This result confirms the expectation that higher order polynomials can describe more complex distortions (Raffel et al. 1998).

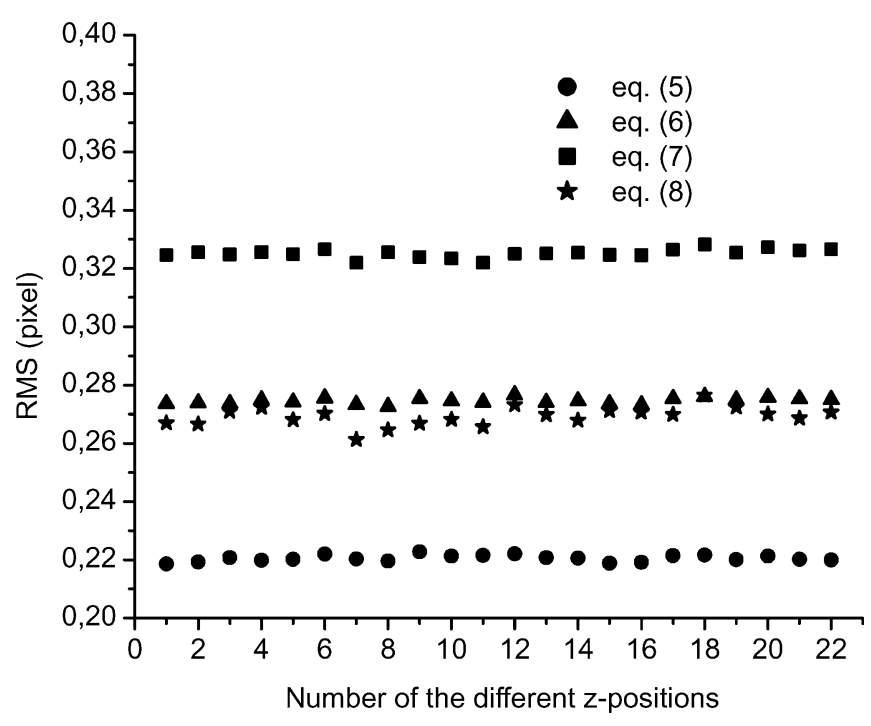

Fig. 3. RMS values of deviation from true coordinates when applying four different mapping functions; abscissa: number value of the different $z$-positions 


\section{3}

\section{Calibration by using an artificial neuronal network}

The higher order polynomials are approaches to the imaging function whose exact form is not known. Under certain circumstances, artificial neuronal networks (ANN) are expected to approach the desired relationship very accurately. Therefore, we find it interesting to check the capabilities of an ANN for establishing a relationship between the coordinates in the image plane and those in the physical plane. For this purpose we make use of the toolbox available with the Matlab 5.0 software. A twolayer feed-forward net with the input variables $\left(X_{i}, Y_{i}\right)$, as coordinates in the recording plane, and the output variables $\left(x_{i}, y_{i}\right)$, as coordinates in the physical plane, is formed in order to provide an approximated imaging function

$\vec{x}=\vec{F}(\vec{X})$

The ANN is applied to and trained with the calibration grid described in Sect. 3.2. From the total of 117 nodal points, the coordinates of 93 points are used for the training of the network, while the coordinates of the remaining 24 nodal points serve for testing the performance of the network after the training. The 24 points for the testing are selected such that they are uniformly and equally distributed in the plane of the grid. Additional information on the training of the ANN and the optimisation of the number of neurons for the approximation process is available in Fei (2002).

The ANN is tested in the same way as reported in Sect. 3.2 for checking the accuracy of the imaging functions: The ANN is applied to the images of the grid shifted to various positions in the water tank for converting the distorted images. The RMS values of the deviations of the predictions from the known true positions of the 24 nodal points at different $z$-positions are shown in Fig. 4, which also includes the results obtained by applying Eq. 5 to these test cases. Obviously, the result from using the ANN is better than that obtained with the third order polynomial. However, a problem with applying the ANN for this

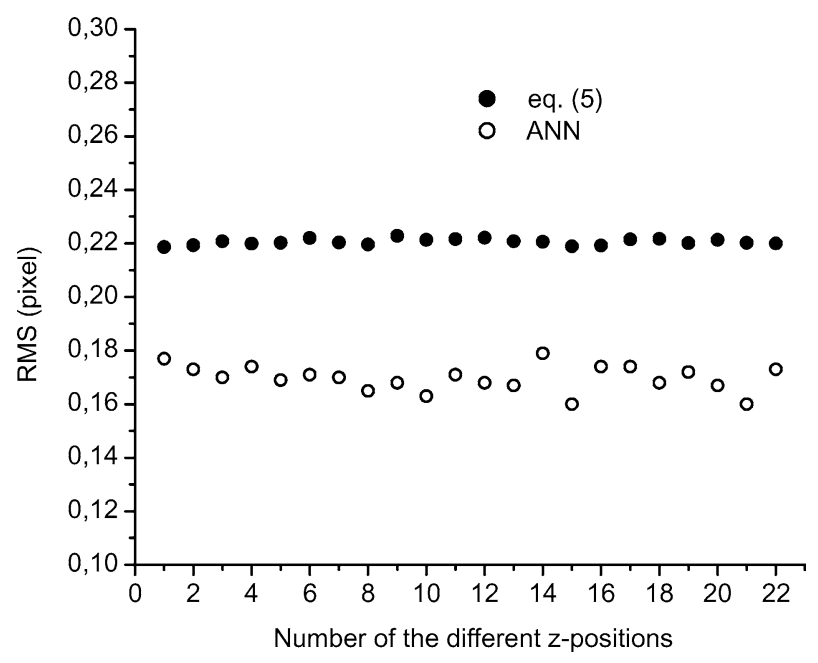

Fig. 4. Comparison of the calibration of a distorted image by applying the third order polynomial, Eq. 5, as imaging function and the ANN purpose is the significantly longer time needed for computation: While the time needed for converting an image of $1280 \times 1024$ pixels in size was only $6 \mathrm{~s}$ using Eq. 5, this computation required $241 \mathrm{~s}$ for the ANN on a Pentium II (233 MHz). The expense of computing time must be considered when deciding about the possible use of an ANN.

\section{4 \\ Experimental verification}

\section{1}

Velocity measurement of a system of particles suspended in water

In this section we report on experimental studies in which the tools developed for a 3D evaluation as described in Sects. 2 and 3 are applied to the motion of particles suspended in water. Several procedures for checking the accuracy of 3D PIV evaluations are reported in the literature, e.g. on the basis of artificially generated PIV patterns (Soloff et al. 1997; Lecerf et al. 1999) or by using as the object an ensemble of particles enclosed in a solid transparent Plexiglas cube (Lawson and Wu 1997). In our experimental check we use a system of particles in the same environment as would occur in liquid flow, i.e. the particles are suspended in water and viewed through the transparent walls of a tank. Subsequently, we evaluate this 3D object with the method of Soloff et al. (1997), which is applicable to this situation.

The object is a Plexiglas tank $200 \mathrm{~mm} \times 200 \mathrm{~mm} \times 50 \mathrm{~mm}$ in size that is filled with water and can be moved at a controlled speed along a straight rail. The thickness of the Plexiglas plane walls is $5 \mathrm{~mm}$. Tracer particles $10 \mu \mathrm{m}$ in size with a specific weight of $1100( \pm 50) \mathrm{kg} / \mathrm{m}^{3}$ are suspended in the water, whose specific weight is adjusted to that of the particles by adding and dissolving an appropriate amount of $\mathrm{NaCl}$. In this way a neutrally buoyant system of particles suspended in the liquid is generated

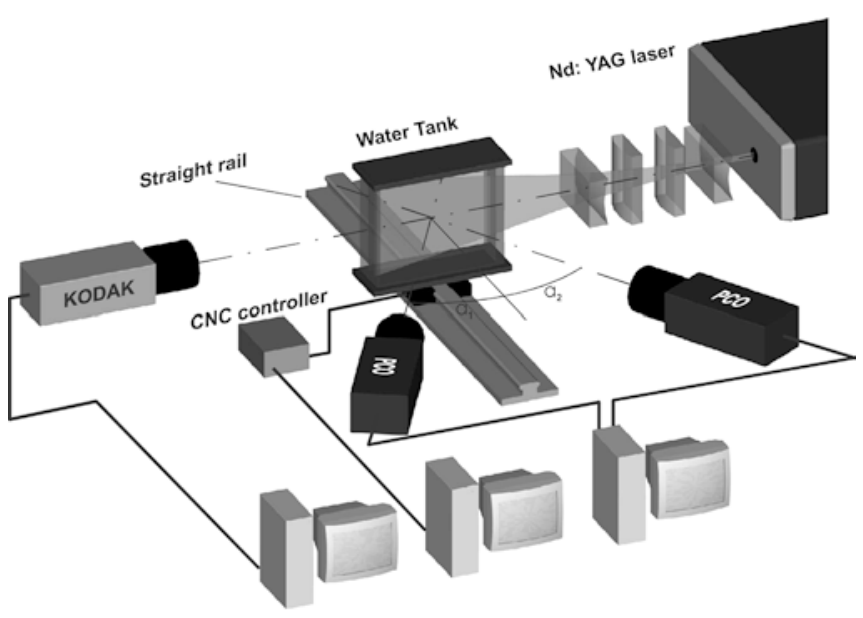

Fig. 5. Experimental set-up for measuring the velocity of the particles within the water tank by the stereo PIV system. The water tank moves along the straight rail, and its instantaneous velocity, as the reference velocity, is measured with a second (2D) PIV system (see the Kodak camera) 
such that the particles assume the velocity of the tank when it is moved with constant speed along the rail. PIV measurements ensured that, after a specific period of acceleration, the velocities of tank, water, and particles are equal.

In our experimental set-up (Fig. 5) the tank, and thus the particles, move in a direction perpendicular to the light sheet, and this velocity had to be determined with the stereo PIV system. The light sheet is generated with a double-pulsed Nd:YAG laser and, in the water, it has a thickness of approximately $1 \mathrm{~mm}$. Two PCO cameras (PCO Flow Master 3, 1280×1024 pixels, LaVision) serve for recording the particle image patterns in the "angular displacement" mode of the stereo system. The Scheimpflug condition is fulfilled by means of special adapters (provided by LaVision) to the cameras. Since the object is moving and the light sheet is fixed in space, the recording of the PIV patterns must be synchronised with the moving tank such that the PIV system is triggered when the tank is at the respective position on the rail. For several reasons the velocity of the tank is not ideally constant, but it must be known as the reference velocity against which the velocity determined with the stereo measurement should be checked. The instantaneous velocity of the tank on the rail is measured with a separate 2D PIV system (see the Kodak camera in Fig. 5 that is directed towards a grid fixed on the side wall of the tank), and thus, the reference velocity is determined and available with the known subpixel accuracy of a 2D PIV measurement.

For the calibration of the stereo system we use a calibration grid immersed in the water tank, with the third order polynomial according to Eq. 5, and we follow the procedure explained in Sect. 3.1. Similarly, the determination of the viewing angles $\alpha_{1}$ and $\alpha_{2}$, needed for using Eq. 1, is done as described in Sect. 2. The particle image patterns are evaluated with the MQD method (Gui and Merzkirch 1996).

The tank is moved on the rail in three different velocity ranges: approximately $75 \mathrm{~mm} / \mathrm{s}, 100 \mathrm{~mm} / \mathrm{s}$, and $150 \mathrm{~mm} / \mathrm{s}$. The actual tank velocity in each experiment is determined with the 2D PIV system and with the accuracy of a 2D PIV measurement. A comparison of the "reference velocity", measured in this way, and the result of the stereo measurement are given in Fig. 6 for the three velocity ranges. The horizontal scale is the reference velocity, while the vertical scale applies to the values measured with the stereo PIV system. The solid line at $45^{\circ}$ indicates complete agreement between the two values, and the two dashed lines on each side of the solid line confine the range of $\pm 3 \%$ relative deviation between reference and stereo measurements. All experimental values lie within this range. For the $75 \mathrm{~mm} / \mathrm{s}$ range, the average of the absolute value of the relative deviation is $1.1 \%$, and the maximal deviation is $2.2 \%$. These values are for the $100 \mathrm{~mm} / \mathrm{s}$ range $1.1 \%$ and $2.8 \%$, respectively, and for the $150 \mathrm{~mm} / \mathrm{s}$ range $1.2 \%$ and $2.7 \%$, respectively. The deviations can be attributed to several independent effects. One possible effect results from the fact that the motion of the tank is normal to the light sheet. This arrangement requires that the time interval between the two YAG laser pulses has to be short enough to ensure that the particles remain within the light sheet during that interval. Therefore, the distance between the particle images is only of the order of 1 to 2 pixels. Assuming a resolution of 0.01 pixels in the
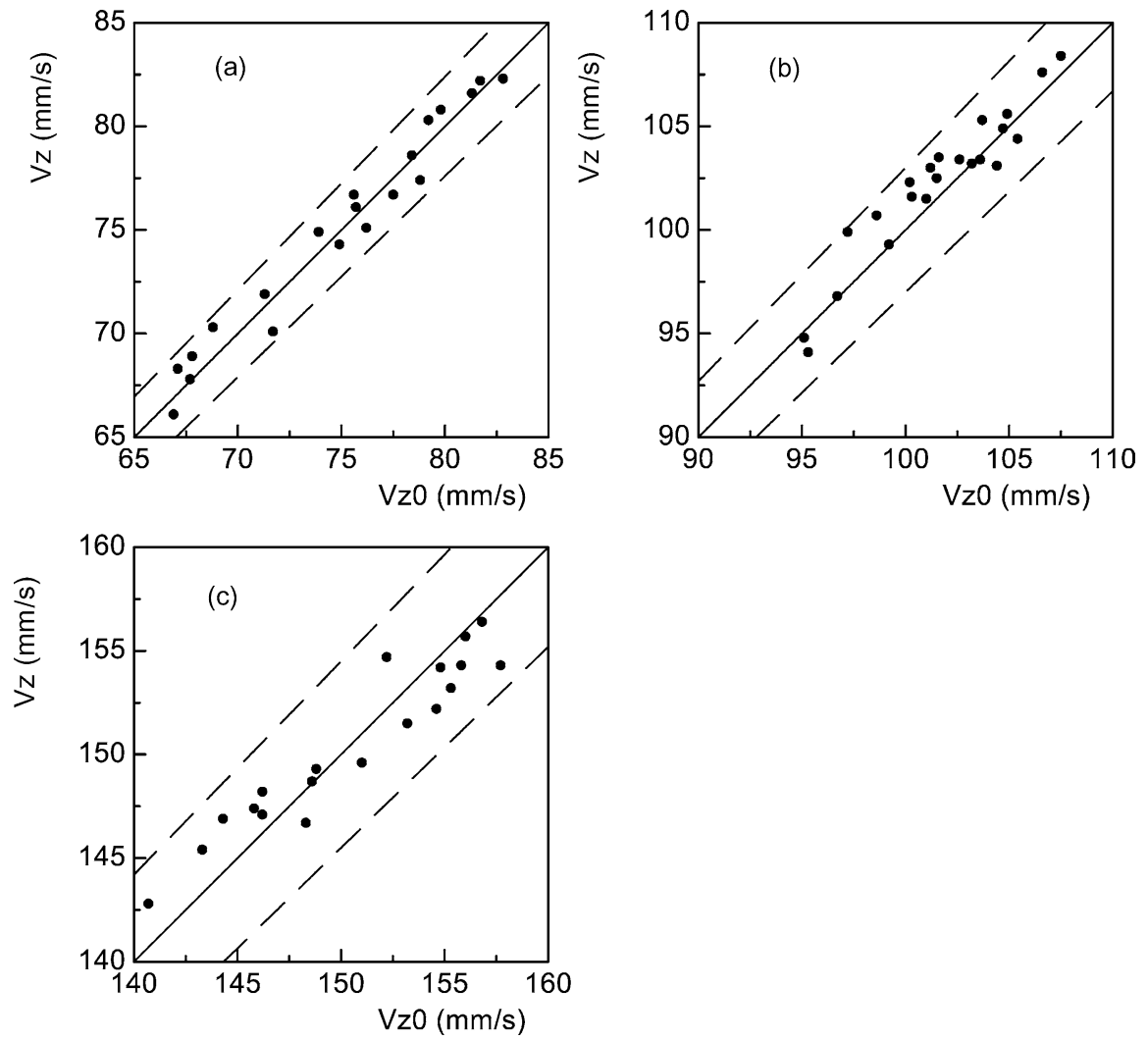

Fig. 6a-c. Comparison of "reference velocity" and velocity measured with the stereo PIV system. Dashed lines indicate $\pm 3 \%$ deviation. Velocity ranges: a $75 \mathrm{~mm} / \mathrm{s} ;$ b $100 \mathrm{~mm} / \mathrm{s} ;$ c $150 \mathrm{~mm} / \mathrm{s}$ 
measurement, the contribution of this effect to the abovementioned deviation is of the order of $1 \%$.

\section{2}

\section{Comparison with the method of Soloff et al. (1997)}

The method of Soloff et al. (1997) uses a three-dimensional polynomial for relating the positions in the $3 \mathrm{D}$ physical space and the respective positions in the $2 \mathrm{D}$ recording planes of the cameras. Therefore it can be applied to the flow of a liquid in a tank where it is necessary to record the particle image patterns through transparent walls. The coefficients of the polynomial are found by calibration. A further difference to the method presented in this paper is that the PIV recordings are evaluated prior to being converted by means of the calibration procedure; the conversion or correction of the geometrical coordinates is done in the planar distribution of the evaluated displacement data. We apply the method of Soloff et al. (1997) to the same object that we have used for verifying the evaluation method presented in this paper (see Sect. 4.1). The necessary steps in this evaluation and the principal differences between the two methods are discussed in detail by Fei (2002).

The comparative studies with the two methods are performed in the same set-up that is used for the calibration (see Sect. 3.2). The grid positioned in the filled water tank is used for both the calibration and checking of the accuracy of the two methods. The arrangement for these studies is sketched in Fig. 7. For determining the viewing angle and calibrating the method described in this paper the grid pattern must be recorded at two different $z$-positions of the grid, here: $z=+0.5 \mathrm{~mm}$ and $-0.5 \mathrm{~mm}$, while three positions are necessary for calibrating the method of Soloff et al.; we use the $z$-coordinates $+0.5 \mathrm{~mm}$, 0 , and $-0.5 \mathrm{~mm}$. All $z$-displacements of the grid are controlled with the 2D PIV system for which the Kodak camera is used (Fig. 7), and these values are taken as a reference. When the tank is displaced between exposures, the optical path from the light sheet to the lens changes slightly. Since this occurs both in the determination of the viewing angles and in the experiment, we assume that this effect is compensated automatically. After the calibration the grid is shifted to several positions near $z=-0.4 \mathrm{~mm}$, and the displacement with regard to the $z=0$ position is

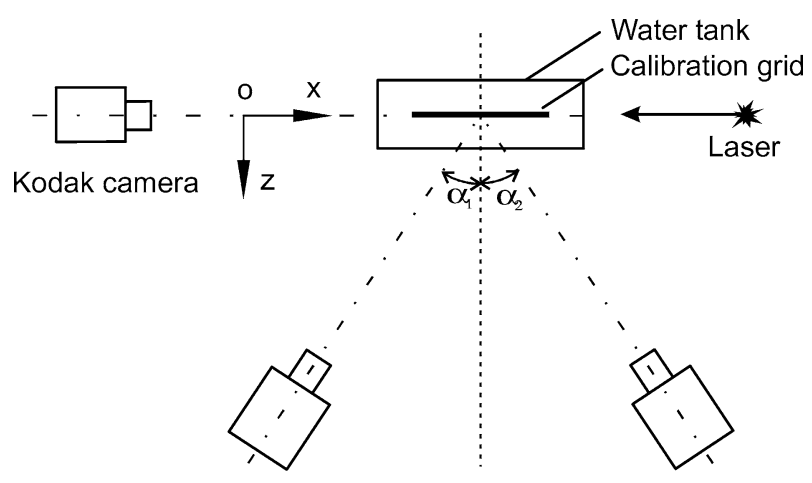

PCO camera 1

PCO camera 2

Fig. 7. Set-up for calibrating the evaluation methods measured by means of the two methods. The results are shown in Fig. 8 where the abscissa is the "true" (reference) value of the displacement, while the ordinate is the displacement measured with the two stereo evaluations. The $45^{\circ}$ line indicates complete agreement of the measurement with the reference value. The experimental points are close to this line, and the difference between the two methods appears to be small.

The difference between the two methods can be recognised easier in the diagram of Fig. 9, where the relative deviations from the reference values are shown for the method presented in this paper (abscissa) and the method of Soloff et al. (ordinate). Apparently, smaller deviations result from the present method; this result is, of course, restricted to this particular experiment.

\section{5}

\section{Conclusion}

In order to perform reliable measurements of the three velocity components in liquid flow with a stereo PIV system it is mandatory to minimise the errors made in determining the $2 \mathrm{C}$ displacement vectors and the viewing direction of each of the two cameras. The difficulties in determining these quantities with high accuracy result from the refraction through the transparent walls confining the liquid. We present a method for determining the viewing direction by using digital imaging procedures such that it becomes unnecessary to directly measure geometrical parameters of the set-up. When applying this method in the reported experiment the viewing angle is just a coefficient, analogous to the coefficients found by calibration. For calibrating the coordinate system in the physical plane against the (distorted) coordinates in the imaging plane we use a third order polynomial that, as one can expect, provides more accurate results than imaging functions of lower order would do. An additional improvement is obtained when the calibration is done by means of an artificial neural network, but at the expense of considerably increasing the computation time. A comparison of the evaluation results obtained with the operational procedures presented in this paper and those

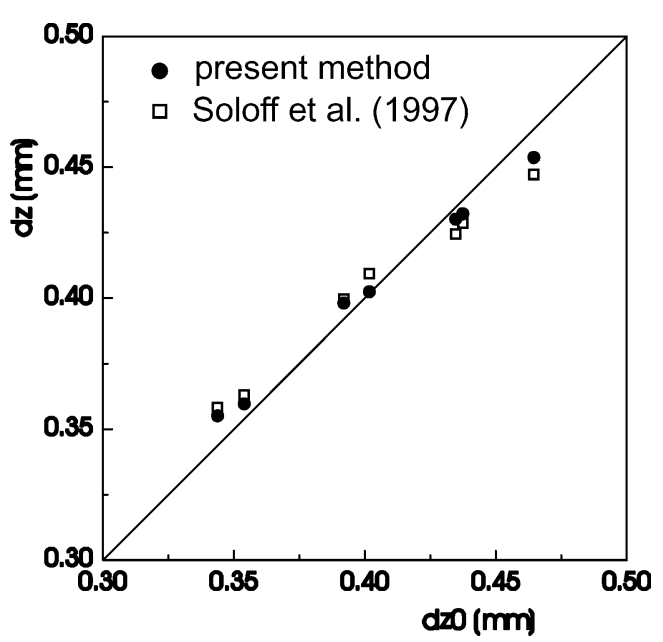

Fig. 8. Comparison of measuring the known displacement of the grid with the two methods 


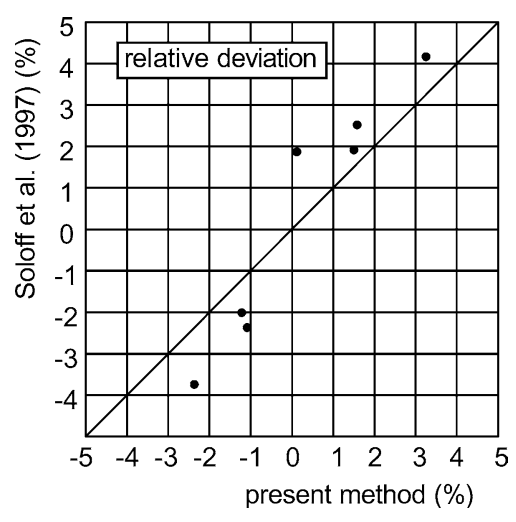

Fig. 9. Relative deviation from the reference values obtained by applying the two methods

generated with another method that is applicable to liquid flow (Soloff et al. 1997) shows, that the presented methods can be considered as viable alternatives to existing methods. In order to account for the imaging through the transparent walls the method of Soloff et al. makes use of a three-dimensional calibration, while for the present method a 2D calibration is sufficient. The algorithms and procedures proposed here can be implemented easily in the respective PIV software.

\section{References}

Coudert S, Schon J-P (2001) Back-projection algorithm with misalignment corrections for 2D3C stereoscopic PIV. Meas Sci Technol 12:1371-1381
Ehrenfried K (2002) Processing calibration-grid images using the Hough transformation. Meas Sci Technol 13:975-983

Fei R (2002) Untersuchungen über die Auswertesicherheit bei der Stereo-Particle-Image-Velocimetry. Dissertation, Universität Essen, Shaker-Verlag, Aachen

Fei R, Gui L, Merzkirch W (1999) Comparitive study of correlationbased PIV evaluation methods. Mach Graph Vision 8:571-578

Gui L, Merzkirch W (1996) A method of tracking ensembles of particle images. Exp Fluids 21:465-468

Hinsch KD (1995) Three-dimensional particle velocimetry. Meas Sci Technol 6:742-753

Jähne B (1997) Digitale Bildverarbeitung. Springer, Berlin Heidelberg, New York

Lawson NJ, Wu J (1997) Three-dimensional particle image velocimetry: Experimental error analysis of a digital angular stereoscopic system. Meas Sci Technol 8:1455-1464

Lecerf A, Renou B, Allano D, Boukhalfa A, Trinité M (1999) Stereoscopic PIV: validation and application to an isotropic turbulent flow. Exp Fluids 26:107-115

Prasad AK (2000) Stereoscopic particle image velocimetry. Exp Fluids 29:103-116

Prasad AK, Adrian RJ (1993) Stereoscopic particle image velocimetry applied to liquid flows. Exp Fluids 15:49-60

Prasad AK, Jensen K (1995) Scheimpflug stereo camera for particle image velocimetry in liquid flows. Appl Opt 34:7092-7099

Raffel M, Willert C, Kompenhans J (1998) Particle image velocimetry. Springer, Berlin Heidelberg, New York

Soloff SM, Adrian RJ, Liu ZC (1997) Distortion compensation for generalized stereoscopic particle image velocimetry. Meas Sci Technol 8:1441-1454

Westerweel J, van Oord J (1999) Stereoscopic PIV measurements in a turbulent boundary layer. In: Stanislas M, Kompenhans J, Westerweel J (eds) Particle image velocimetry: Progress toward industrial application. Kluwer, Dordrecht

Willert C (1997) Stereoscopic digital particle image velocimetry for application in wind tunnel flows. Meas Sci Technol 8:1465-1479 